\title{
Drug-drug interaction between cannabidiol and phenobarbital in healthy dogs
}

Caitlin E. Doran, VMD ${ }^{1 *}$; Stephanie McGrath, MS, DVM ${ }^{1}$; Lisa R. Bartner, MS, DVM ${ }^{1}$; Breonna Thomas, MS ${ }^{1}$; Alastair E. Cribb, DVM, $\mathrm{PhD}^{2}$; Daniel L. Gustafson, $\mathrm{PhD}^{1}$

\author{
'Department of Clinical Sciences, College of Veterinary Medicine and Biomedical Sciences, Colorado State University, Fort Collins, CO \\ ${ }^{2}$ Department of Biomedical Sciences, Cummings School of Veterinary Medicine at Tufts University, Grafton, MA \\ *Corresponding author: Dr. McGrath (stephanie.mcgrath@colostate.edu)
}

\section{OBJECTIVE}

To assess drug-drug interactions between cannabidiol (CBD) and phenobarbital (PB) when simultaneously administered to healthy dogs.

\section{ANIMALS \\ 9 healthy, purpose bred Beagles.}

\section{PROCEDURES}

A 3-phase prospective, randomized pharmacokinetic (PK) interaction study of CBD and PB was performed as follows: phase I, CBD PK determination and evaluation of CBD tolerability by 3 single-dose CBD (5 mg/kg, $10 \mathrm{mg} / \mathrm{kg}$, and $20 \mathrm{mg} / \mathrm{kg}) \mathrm{protocols}$ followed by 2-week CBD dosing; phase 2, a single-dose, 3-way, crossover PK study of CBD (10 mg/kg), PB (4 mg/kg), or CBD $(10 \mathrm{mg} / \mathrm{kg})$ administration plus PB (4 mg/kg); and phase 3, evaluation of chronic PB (4 mg/kg, q $30 \mathrm{~d})$ administration followed by single-dose CBD (10 mg/kg) PK study.

\section{RESULTS}

Although there were variations in CBD PK variables in dogs receiving CBD alone or in conjunction with $\mathrm{PB}$, significance differences in CBD PK variables were not found. No significant difference was observed in PB PK variables of dogs receiving PB alone or with CBD. During chronic CBD administration, mild gastrointestinal signs were observed in 5 dogs. At daily CBD doses of 10 to $20 \mathrm{mg} / \mathrm{kg} / \mathrm{d}$, hypoxia was observed in 5 dogs and increased serum alkaline phosphatase (ALP) activities (range, $30 \mathrm{I}$ to 978 $\mathrm{U} / \mathrm{L}$ ) was observed in 4 dogs. A significant increase in ALP activity was observed with chronic administration of CBD during phase I between day 0 and day 14 .

\section{CLINICAL RELEVANCE}

No significant PK interactions were found between CBD and PB. Dose escalation of CBD or adjustment of PB in dogs is not recommended on the basis of findings of this study.

$\mathrm{E}^{\mathrm{a}}$ pilepsy affects $0.5 \%$ to $5.7 \%$ of the canine population, making it one of the most common neurologic conditions faced in veterinary medicine. ${ }^{1}$ Currently, there are only limited numbers of licensed antiepileptic drugs (AEDs) available for use for seizure management in dogs. The American College of Veterinary Internal Medicine consensus statement recommends phenobarbital (PB) and potassium bromide as first-line medications. ${ }^{2}$ However, a combination of these medications is unsuccessful in controlling seizures in approximately $20 \%$ to $30 \%$ of epileptic dogs. ${ }^{3,4}$ The limited availability of safe and highly effective AEDs has led to the search for alternatives. ${ }^{1}$

Cannabis products have gained popularity in recent years as anecdotal evidence of their efficacy as an AED in both animals and humans has been reported. ${ }^{5-11}$ The use of cannabidiol (CBD), a nonpsychotropic phytocannabinoid, has been shown to have both in vitro and in vivo anticonvulsant properties in seizure models, and recently, the FDA approved the use of a 99\% pure CBD oral product to treat severe forms of pediatric epilepsy.,6,9,10 Although not legally prescribable in veterinary medicine, research has begun in CBD's potential utility as an AED. A double-blinded, placebocontrolled pilot study in dogs utilizing CBD in addition to standard AEDs reported an overall reduction in seizure activity in the treatment group, compared with the placebo group, although only 2 dogs in the treatment group were considered responders (greater than fifty percent reduction in seizure frequency). ${ }^{2}$ The nonpsychotropic nature, potential anticonvulsive properties, and high safety profile have led to increased CBD use and its potential of becoming a new AED in both human and veterinary medicine.

Cannabidiol is metabolized by cytochrome $\mathrm{P} 450$ (CYP450) hepatic enzymes and is known to inhibit 
several isoenzymes in humans, including CYP450 2C19, CYP450 2D6, CYP450 2C9, and CYP450 3A4. 2,6,10,12-14 The CYP450 system is involved in the metabolism of many medications, including most AEDs, such as PB. In humans and dogs, PB is metabolized by the CYP450 system, specifically inducing isoenzymes CYP450 2C9 and CYP450 3A4 in humans and CYP450 2B, CYP450 2C, and CYP450 3A in a variety of species. ${ }^{3,6}$ Pharmacokinetic (PK) interactions between AEDs and CBD have not been previously studied in dogs, but in humans, a recent study reported a significant effect on clobazam, topiramate, rufinamide, eslicarbazepine and zonisamide in adults. ${ }^{14}$ In vitro $\mathrm{CBD}$ PK studies have shown that CBD coadministered with other medications utilizing the CYP450 pathways, such as rifampicin, which, similar to $\mathrm{PB}$, is a CYP3A4 inducer, resulted in reduced peak plasma concentrations of CBD. ${ }^{15}$ As such, it is reasonable to speculate that CBD may affect AED PK and vice versa when used in canine patients. Despite lack of FDA approval and that veterinarians cannot legally authorize use, there is increasing CBD use by the general public in their pets. As such, a controlled drug-drug interaction study between CBD and PB is needed. This study aims to assess for potential drugdrug interaction between PB and CBD in healthy Beagles. We hypothesize that CBD, when simultaneously orally administered with $\mathrm{PB}$, will lead to $\mathrm{PK}$ alterations in 1 or both drugs.

\section{Materials and Methods}

\section{Animals}

All aspects of the study were approved by the Colorado State University's Institutional Animal Care and Use Committee (protocol ID 19-8683A, approved April 24, 2019). Nine healthy, purpose bred, female sexually intact Beagles, between 4 and 5 years of age were enrolled. Dogs weighed a mean of $9.5 \mathrm{~kg}$ (range, 8.4 to $11.1 \mathrm{~kg}$ ). Each dog underwent routine laboratory testing (CBC determination chemistry panel analysis) and physical examination by a veterinarian prior to enrollment. All animals were kept in an on-site research facility and were attended to daily by Laboratory Animal Resource staff or veterinarians for feeding, cleaning, and evaluation of overall appearance. Because 1 dog had to be housed separately because of intergroup aggression, the dogs were separated into 2 groups of 3 dogs, 1 group of 2 dogs, and 1 dog housed alone. Each dog was identified by distinct ear tattoos. Dogs were fed a commercial diet (Hill's Science Diet Chicken \& Barley Adult wet food or Exclusive Adult Dog Chicken \& Brown Rice Formula dry food) throughout the study time. All examination findings were recorded throughout the study.

\section{Study design}

A 3-phase randomized prospective study was designed (Figure I).
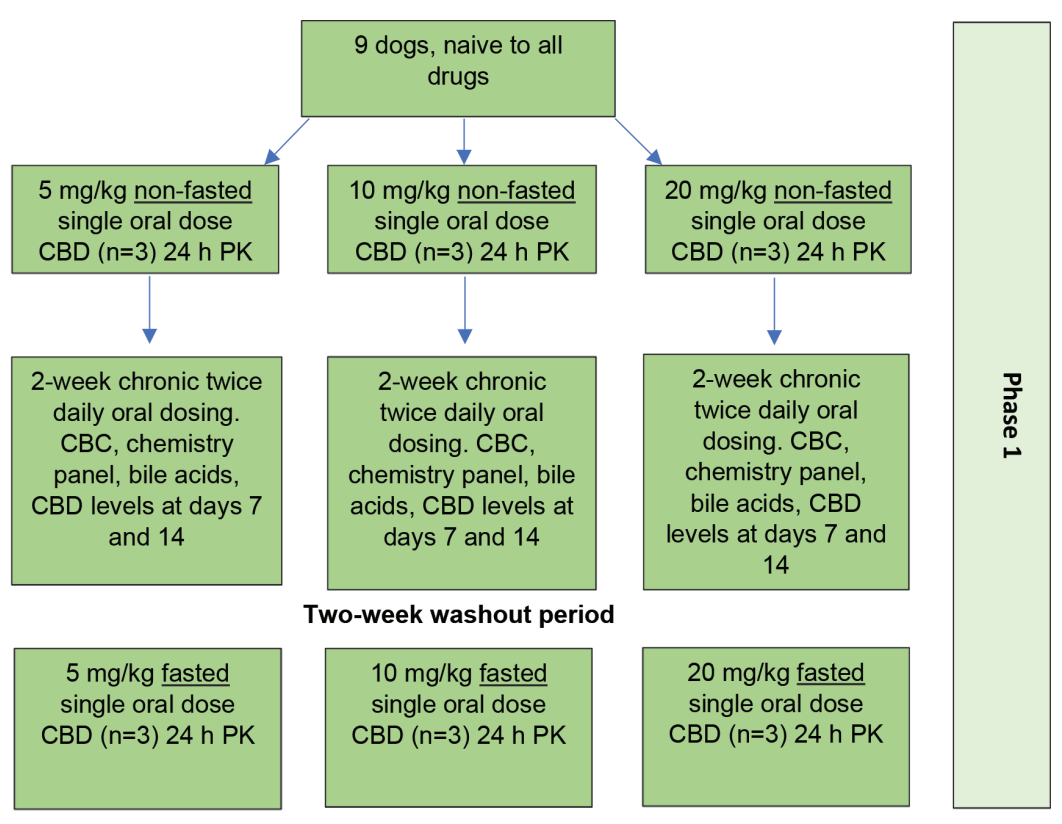

Two-week washout period

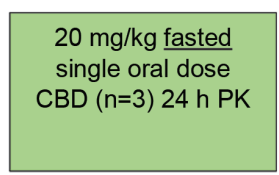

9 dogs, randomly assigned and rotated through each of 3 groups ( $n=3 /$ group)

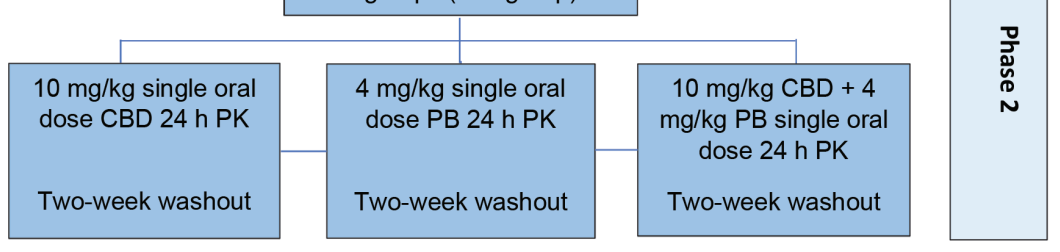

Two-week washout period

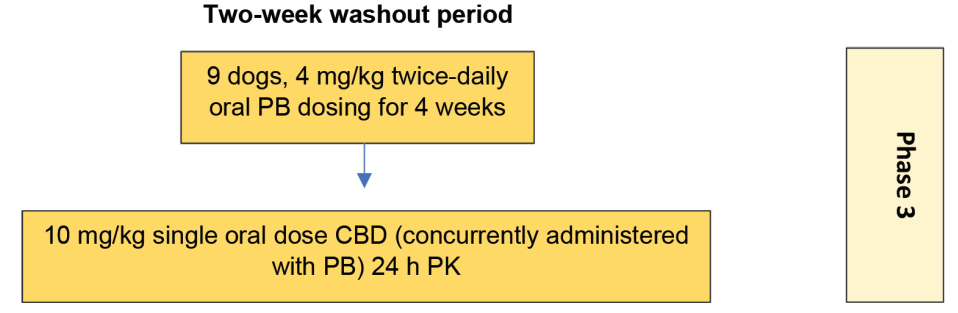

Figure I-Flow diagram of study design broken into the 3 phases of the study. Phase I includes random assignment of 9 dogs into I of 3 cannabidiol (CBD) dosing groups. In phase 2 , the same 9 dogs following a washout period were randomly assigned to I of 3 groups: CBD (10 mg/kg), phenobarbital (PB; $4 \mathrm{mg} /$ $\mathrm{kg})$, or CBD-PB ( $10 \mathrm{mg} / \mathrm{kg}$ and $4 \mathrm{mg} / \mathrm{kg}$, respectively) and after a 2 -week washout were randomly assigned to the other treatment groups they had not received yet. During phase 3 , all 9 dogs received chronic PB dosing $(4 \mathrm{mg} / \mathrm{kg})$ for 4 weeks and a single CBD dose $(10 \mathrm{mg} / \mathrm{kg})$ on day 30. 


\section{Phase I-CBD PK study}

Dogs were randomly assigned to 1 of 3 groups of $\mathrm{CBD}$ doses. During this phase, each dog received a single dose of highly purified CBD isolate (100\% pure) in a medium chain triglyceride (MCT) oil base (CBD in MCT; Canopy Growth Corporation). Cannabidiol purity was verified by analysis (PhytaTech Metric Solutions). Group $1(\mathrm{n}=3)$ received a CBD dose of $5 \mathrm{mg} / \mathrm{kg}$, PO; group $2(\mathrm{n}=3)$ received a CBD dose of $10 \mathrm{mg} / \mathrm{kg}, \mathrm{PO}$; and group $3(\mathrm{n}=3)$ received a CBD dose of $20 \mathrm{mg} / \mathrm{kg}$, PO. Doses for the CBD dosing groups was chosen based on results of a prior study. ${ }^{16}$ Blood was collected from the jugular vein at times 0 , $2,4,8,12$, and 24 hours with physical examinations being performed prior to each blood sample collection by veterinarians or veterinary students.

At time 0 hour, $9 \mathrm{~mL}$ of blood was collected from each dog and placed in EDTA tube ( $2 \mathrm{~mL})$ for a CBC, red top tube $(3 \mathrm{~mL})$ for chemistry panel analysis and fasted bile acids test, and 2 sodium heparin tubes ( 2 $\mathrm{mL}$ each) for CBD plasma concentration determination. The tubes were placed on ice until all samples were collected. The sodium heparin tubes were centrifuged at $2,000 \mathrm{Xg}$ at $4{ }^{\circ} \mathrm{C}$ for 10 minutes. The plasma was separated into cryotubes and stored at -80 ${ }^{\circ} \mathrm{C}$ until processing. Blood samples were processed within 1.5 hours of initial sample collection time. Following time 0 hour for blood collection, all dogs were fed 2 tablespoons of wet dog food (Hill's Science Diet Chicken \& Barley Adult wet food or Exclusive Adult Dog Chicken \& Brown Rice Formula dry food) and immediately administered their respective CBD dose. Dosing amount and time were recorded.

At time 2 hours, $7 \mathrm{~mL}$ of blood was collected from the jugular of each dog with $3 \mathrm{~mL}$ placed in a red top tube for postprandial bile acids testing and $2 \mathrm{~mL}$ each placed into 2 sodium heparin tubes for CBD plasma concentration determination. Sodium heparin tubes were processed as already stated.

At times $4,8,12$, and 24 hours, $4 \mathrm{~mL}$ of blood were collected and placed into 2 sodium heparin tubes ( $2 \mathrm{~mL}$ each) and processed for CBD plasma concentration determination.

All dogs continued to receive a twice daily oral dose of CBD as determined by their previously assigned groups (ie, group $1,5 \mathrm{mg} / \mathrm{kg}, \mathrm{q} 12 \mathrm{~h}$; group 2 , $10 \mathrm{mg} / \mathrm{kg}$, q $12 \mathrm{~h}$; and group 3, $20 \mathrm{mg} / \mathrm{kg}$, q $12 \mathrm{~h}$ ) for a total of 14 days during which time daily physical examinations were performed and adverse events (AEs) were recorded. Complete blood count determination, chemistry panel analysis, bile acids test (preprandial and 2-hours postprandial), and CBD concentration determination were performed on days 7 and 14 .

To compare nonfasted and fasted CBD PK findings, following a 2-week washout period, the dogs received another single dose of the same pure CBD isolate PO (based on their previously determined group) after being fasted for at least 12 hours. Blood was collected at time $0,2,4,8,12$, and 24 hours and processed as already described.

\section{Phase 2-Single-dose CBD and PB interaction}

All dogs underwent a 2-week washout period prior to the initiation of phase 2, a single-dose, 3-way, crossover study. Dogs were randomly assigned into 1 of 3 oral treatment groups as follows: 1) CBD-infused MCT oil $(10 \mathrm{mg} / \mathrm{kg}$ ); 2) PB tablets (Phenobarbital tablets; Winder Labs; $4 \mathrm{mg} / \mathrm{kg})$; or 3) CBD-PB (10 mg/kg and 4 $\mathrm{mg} / \mathrm{kg}$, respectively). Blood was collected at time 0,2 , $4,8,12$, and 24 hours as previously described for CBC determination and chemistry panel analysis at time 0 , and $\mathrm{CBD}$ and/or PB concentration determination at all time points. Blood for determination PB concentrations $(2 \mathrm{~mL})$ was placed in red top tubes and centrifuged at $2,500 \mathrm{X} g$ for 10 minutes. The serum was separated into a red top tube and refrigerated until processed (within 1.5 hours of sample collection time). The plasma for $\mathrm{CBD}$ analysis was stored at $-80^{\circ} \mathrm{C}$. Each dog underwent all 3 drug administration protocols with a 2-week washout period between each drug exposure.

\section{Phase 3-Chronic PB administration}

After a 2-week washout period, all dogs received twice daily PB administration ( $4 \mathrm{mg} / \mathrm{kg}, \mathrm{PO}$ ) for a total of 4 weeks. Dogs were evaluated daily. At the end of 4 weeks, all dogs were administered a single oral dose of CBD $(10 \mathrm{mg} / \mathrm{kg})$ with the $4 \mathrm{mg} / \mathrm{kg}$ dose of PB and blood was collected at times $0,2,4,8,12$, 24 hours for CBC determination, chemistry panel analysis, and measurements of $\mathrm{PB}$ concentrations and $\mathrm{CBD}$ concentrations as previously described. Adverse events were recorded throughout the study period.

\section{Laboratory analysis of blood samples}

Phenobarbital concentrations were analyzed through the Colorado State University Center for Companion Animal Studies via chemiluminescent immunoassay (Immulite 1000; Siemens Medical Systems USA). Cannabidiol was measured in canine plasma with a validated liquid chromatography-mass spectrometry-mass spectrometry assay (Instrumentation included an Applied Biosystems 3200 Q-TRAP triple quadrupole mass spectrometer coupled to a Shimadzu LC20AD and HTC-PAL autosampler) performed in the Pharmacology Laboratory of the Drug Development \& Discovery Shared Resource (University of Colorado Cancer Center). ${ }^{2}$ Additional information regarding the assay methods are available (Supplementary Appendix SI). Complete blood count, chemistry panel analysis, and bile acids tests were performed by the Colorado State University Veterinary Clinical Pathology Laboratory.

\section{Statistical analysis}

Pharmacokinetic parameters were calculated by noncompartmental analysis with computer software (Excel version 2005; Microsoft Corp).$^{17}$ Parameters analyzed included maximal concentration $\left(\mathrm{C}_{\max }\right)$, time to $\mathrm{C}_{\max }\left(\mathrm{T}_{\max }\right), \lambda$, area under the curve (AUC), half-life, and apparent clearance $(\mathrm{Cl} / \mathrm{F})$ for $\mathrm{CBD}$ dur- 
Table I-Phase I mean area under the curve $\left(A \cup C_{0-t}\right)$, maximal concentration $\left(C_{\text {max }}\right)$, half-life, and clearance $(\mathrm{Cl} / \mathrm{F})$ for 3 cannabidiol (CBD) dosing groups in healthy dogs.

\begin{tabular}{llcccc} 
CBD Dose & & $\begin{array}{c}\text { AUC } \mathbf{0}_{\mathbf{t}} \text { mean } \pm \text { SD } \\
(\mathbf{n g} / \mathbf{m L} \cdot \mathbf{h r})\end{array}$ & $\begin{array}{c}\mathbf{C}_{\max } \text { mean } \pm \text { SD } \\
(\mathbf{n g} / \mathbf{h})\end{array}$ & Half-life (hr) & $\begin{array}{c}\text { Cl/F mean } \pm \text { SD } \\
(\mathbf{n g} / \mathbf{h})\end{array}$ \\
\hline $5 \mathrm{mg} / \mathrm{kg}$ & Fasted & $1130.1 \pm 712.1$ & $143.0 \pm 112.1$ & $13.4 \pm 4.4$ & $7.1 \pm 6.6$ \\
& Nonfasted & $1977.1 \pm 1389.4$ & $581.0 \pm 400.9$ & $19.3 \pm 7.7$ & $5.2 \pm 5.9$ \\
$10 \mathrm{mg} / \mathrm{kg}$ & Fasted & $1370.5 \pm 671.4$ & $231.2 \pm 222.6$ & $6.5 \pm 2.2$ & $8.3 \pm 3.2$ \\
& Nonfasted & $3215.9 \pm 1196.0$ & $579.0 \pm 150.0$ & $7.5 \pm 3.5$ & $3.4 \pm 1.1$ \\
$20 \mathrm{mg} / \mathrm{kg}$ & Fasted & $1289.0 \pm 638.1$ & $155.4 \pm 78$ & $8.8 \pm 2.2$ & $19.3 \pm 11.9$ \\
& Nonfasted & $4247.8 \pm 6203.8$ & $288.5 \pm 359.6$ & $11.0 \pm 2.1$ & $21.4 \pm 18.1$ \\
\hline
\end{tabular}

Mean $\mathrm{AUC}_{0-\mathrm{t}}, \mathrm{C}_{\max }$, half-life, and $\mathrm{Cl} / \mathrm{F}$ for the $3 \mathrm{CBD}$ single oral dosing groups in both a fasted and nonfasted state. $\mathrm{N}=3$ for each group.

ing all phases, as well as for PB during phases 2 and 3. Half-life was calculated for the 8,12 , and 24 hour time points where a linear decline in levels was observed across this span when $\log [\mathrm{CBD}]$ was plotted versus time. For the animals in which there was no linear decline over this period, $\lambda$ and resulting half-life were not calculated.

To compare AUC and $\mathrm{C}_{\max }$ between fasted and nonfasted states during phase 1 , the nonparametric Wilcoxon signed rank test was used, because data were not normally distributed when combined across the dose groups. The Spearman rank order correlation test was used to determine whether there was a correlation between CBD dose and AUC or $\mathrm{C}_{\max }$ in phase 1. Paired $t$ test of AUC values between CBD and CBD-PB as well as CBD-chronic PB groups were performed. The Dunnett multiple comparisons test was performed between groups with $\alpha$ set at 0.05 . The ROUT method, a method based on the false discovery rate (FDR), was performed to determine outliers in AUC values with a $\mathrm{Q}$ (maximum desired FDR) set to $0.2 \% .{ }^{18} \mathrm{~A}$ paired $t$ test was also performed on AUC of PB single dose administration and PB single dose with coadministration of CBD.

Analysis of serum alkaline phosphatase (ALP) activity change during phase 1 was performed with computer software (R Software R 3.6.2; R Core Team 2020). A mixed model was fit with $\log [$ ALP] as the response. Log transformation was used to satisfy model assumptions. Fixed effects included time $(0,7$ or 14 days) and dose $(5,10$, or $20 \mathrm{mg} / \mathrm{kg})$ and time*dose interaction. The Dunnett method was used to compare downstream time points versus baseline for each dose. The Tukey method was used to compare between doses at each time point. For all comparisons, a value of $P$ $<0.05$ was considered significant.

\section{Sample size calculation}

As the variability in PB clearance is approximately $30 \%$ in dogs, ${ }^{19-21}$ a sample population of 8 dogs would allow for detection of a $40 \%$ change in exposure based
A
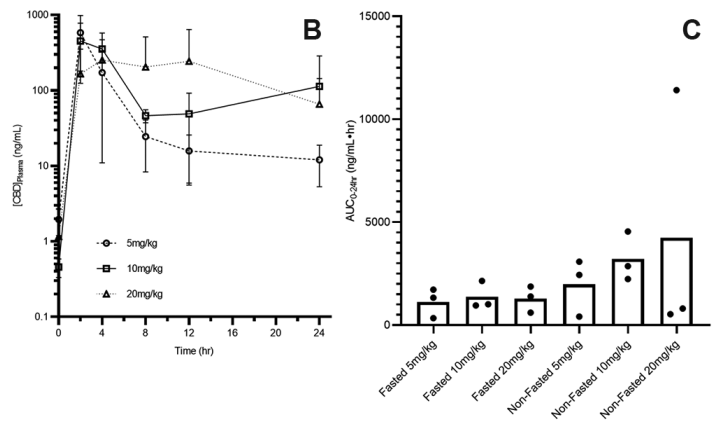

C

Figure 2-A-Concentration versus time curve of mean plasma CBD $(\mathrm{ng} / \mathrm{mL})$

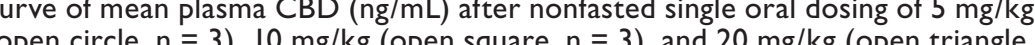
both nonfasted and fasted state with $n=3$ in each group. Individual AUC for each group represented by dots.

on a power of $76 \%$ with 1-way ANOVA pairwise, 2 -sided equality test. Therefore, to achieve a power of greater than $80 \%, 9$ dogs were needed.

\section{Adverse Events}

Adverse events were monitored and graded based on the 2011 consensus document from the Veterinary Cooperative Oncology Group. ${ }^{22}$ Grade 1 AEs consist of mild clinical signs not requiring intervention; grade 2 AEs consist of moderate signs requiring noninvasive intervention that only moderately limits daily activities; grade 3 AEs consist of severe medically important clinical signs that are not life threatening; grade 4 AEs consist of life threatening signs that require urgent intervention; and grade 5 AEs consist of death related to the AE. ${ }^{22}$

\section{Results}

\section{CBD PK results}

Of the 9 dogs enrolled in the study, all dogs successfully completed the study. Cannabidiol data from 1 dog were excluded from phase 3 (CBD-chronic PB) following ROUT method to determine outliers, leaving data from 8 of 9 dogs to be analyzed for the effects of chronic PB administration on CBD PK variables. Plasma CBD concentrations were determined at 5 time 
Table 2-Phases 2 and 3 mean, median, 95\% Cl, minimum and maximum for AUC0-t , Cmax , half-life, and $\mathrm{Cl} / \mathrm{F}$ for 3 states of $\mathrm{CBD}$ administration.

\begin{tabular}{|c|c|c|c|c|c|c|}
\hline \multirow[b]{2}{*}{ PK Parameters } & \multicolumn{6}{|c|}{ CBD } \\
\hline & Mean & SD & Median & $95 \% \mathrm{Cl}$ & Min & Max \\
\hline $\mathrm{C}_{\max }(\mathrm{ng} / \mathrm{mL})$ & 165.1 & 86.2 & 204.0 & 66.3 & 65.8 & 263.0 \\
\hline Half-life (hr) & 9.4 & 5.8 & 12.7 & 5.388 & 5.2 & I7.1 \\
\hline$A \cup C_{0-t}$ & 1024.4 & 623.0 & 1103.3 & 478.9 & 501.4 & 2120.7 \\
\hline \multirow[t]{2}{*}{$\mathrm{Cl} / \mathrm{F}(\mathrm{L} / \mathrm{h} / \mathrm{kg})$} & 9.9 & 6.4 & 8.3 & 4.9 & 4.7 & 19.9 \\
\hline & \multicolumn{6}{|c|}{ CBD / PB } \\
\hline $\mathrm{C}_{\max }(\mathrm{ng} / \mathrm{mL})$ & 154.0 & 109.0 & 145.5 & 83.8 & 33.1 & 347.0 \\
\hline Half-life (hr) & 6.3 & 3.2 & 6.6 & 3.4 & 6.4 & 10.9 \\
\hline $\mathrm{AUC}_{0-\mathrm{t}}$ & I 272.7 & 945.7 & 896.3 & 751.9 & 394.4 & 3433.8 \\
\hline \multirow[t]{2}{*}{$\mathrm{Cl} / \mathrm{F}(\mathrm{L} / \mathrm{h} / \mathrm{kg})$} & 10.5 & 7.8 & 9.4 & 6.0 & 2.9 & 25.4 \\
\hline & \multicolumn{6}{|c|}{ CBD / Chronic PB } \\
\hline $\mathrm{C}_{\max }(\mathrm{ng} / \mathrm{mL})$ & 292.5 & 472.9 & 167.5 & 363.5 & 68.4 & 1620.0 \\
\hline Half-life (hr) & 8.1 & 3.9 & 8.2 & 3.0 & 6.3 & 15.3 \\
\hline$A \cup C_{0-t}$ & 798.8 & 428 & 645.2 & 1646.5 & 336.5 & 7323.3 \\
\hline $\mathrm{Cl} / \mathrm{F}(\mathrm{L} / \mathrm{h} / \mathrm{kg})$ & 13.0 & 9.7 & 12.5 & 7.5 & 1.4 & 29.7 \\
\hline
\end{tabular}

Pharmacokinetic parameters for $\mathrm{AUC}_{0-\mathrm{t}}, \mathrm{C}_{\max }$, estimated elimination half-life, and $\mathrm{Cl} / \mathrm{F}$ for $10 \mathrm{mg} / \mathrm{kg} \mathrm{CBD}$ alone, in conjunction with $4 \mathrm{mg} / \mathrm{kg}$ phenobarbital (CBD-PB), and after 30 days chronic phenobarbital administration at $4 \mathrm{mg} / \mathrm{kg}$ twice daily (CBD-chronic PB). $\mathrm{N}=9$ for all except CBD-chronic PB in which data from I dog ( $\operatorname{dog} 7)$ was excluded as an outlier via ROUT method.

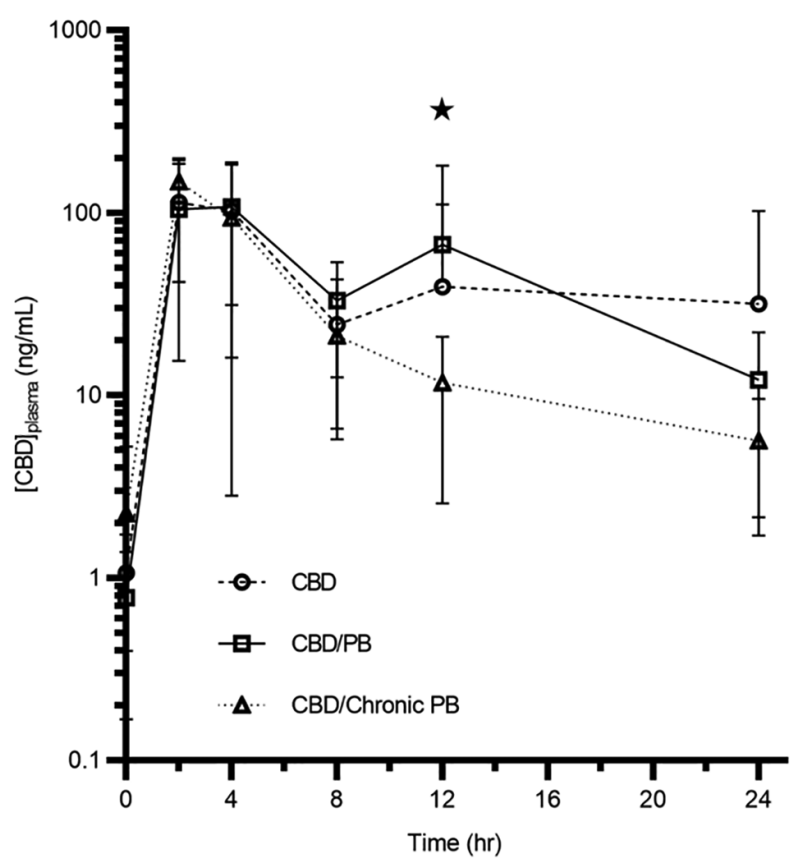

Figure 3-Mean plasma CBD $(\mathrm{ng} / \mathrm{mL})$ concentration versus time (h) from phase 2 (CBD-PB single-dose 3-way crossover pharmacokinetic $[P K]$ ) and phase 3 (CBD-chronic PB PK) in healthy dogs. Cannabidiol $(n=9$, open circle) at $10 \mathrm{mg} / \mathrm{kg}$ single oral dose; CBD-PB ( $\mathrm{n}=9$, open square) at $10 \mathrm{mg} / \mathrm{kg}$ single oral dose $C B D$ and $4 \mathrm{mg} / \mathrm{kg}$ single-dose PB; CBD-chronic PB ( $\mathrm{n}=$ 8 , open triangle) at $4 \mathrm{mg} / \mathrm{kg}$ oral twice daily 30 -day dosing $P B$ with a single $10 \mathrm{mg} / \mathrm{kg} C B D$ dose on day 30 (concurrent with $\mathrm{PB}$ ). Star represents location of second CBD peak, which is not apparent in the CBD-chronic PB group.

points over 24 hours after the initial CBD dose in all 3 phases of the trial. Individual dog data for phase 1 is available (Supplementary table SI), as well as for phases 2 and 3 (Supplementary table S2).
Mean $\pm \mathrm{SD}$, median, $95 \% \mathrm{CI}$, and range for $\mathrm{AUC}_{0}$ t, $\mathrm{C}_{\max }$, half-life, and $\mathrm{Cl} / \mathrm{F}$ for all 3 dosing groups $(5$ $\mathrm{mg} / \mathrm{kg}, 10 \mathrm{mg} / \mathrm{kg}$, and $20 \mathrm{mg} / \mathrm{kg}$ ) of phase 1 (both nonfasted and fasted) are available (Table I; Figure 2). There was no significant difference in the $\mathrm{C}_{\max }$ or the $\mathrm{AUC}_{0-\mathrm{t}}$ between dose, nor any overall correlation with dose, in either the fasted or nonfasted state $\left(P>0.1\right.$ in all cases). The $\mathrm{AUC}_{0-\mathrm{t}}$ was not significantly different between fasted and nonfasted animals $(P=0.14)$. However, the $C_{\max }$ was significantly higher in the nonfasted animals, compared with the fasted animals $(P=0.03)$. The number of animals at each dose was too small to assess for a separate dose effect.

An AUC data point from $1 \mathrm{dog}$ for the CBD-chronic PB group was found to be an outlier after the ROUT method was performed. This data point was excluded from $\mathrm{PK}$ analysis for phases 2 and 3. Mean $\pm \mathrm{SD}$, median, 95\% CI, and range for $\mathrm{AUC}_{0-\mathrm{t}}, \mathrm{C}_{\max }$, half-life, and $\mathrm{Cl} / \mathrm{F}$ for phases 2 and 3 are available (Table 2). The mean $\mathrm{AUC}_{0-\mathrm{t}}$ of single-dose CBD was 1,024.4 \pm 623.0 $\mathrm{ng} / \mathrm{mL} \cdot \mathrm{h}, \mathrm{CBD}-\mathrm{PB}$ combination was $1,272.7 \pm 945.7$ $\mathrm{ng} / \mathrm{mL} \cdot \mathrm{h}$, and CBD-chronic PB combination was 789.8 $\pm 428 \mathrm{ng} / \mathrm{mL} \cdot \mathrm{h}$ (Figure 3 ); there was no significant difference found between mean $\mathrm{AUC}_{0-\mathrm{t}}$ of $\mathrm{CBD}$ administration alone, compared with that from CBD-PB or with CBD-chronic $\mathrm{PB}(P=0.63$ and 0.20 , respectively). The Dunnett multiple comparisons test revealed no significant difference in mean $\mathrm{AUC}_{0-\mathrm{t}}$ between the CBD alone and CBD-PB or with CBD-chronic PB groups (adjusted $P=0.84$ and 0.31 , respectively). In evaluating the concentration over time curves in all 3 groups, a second peak was visible in the CBD and CBD-PB groups, however, a second peak for the CBDchronic PB group was not observed (Figure 4). 

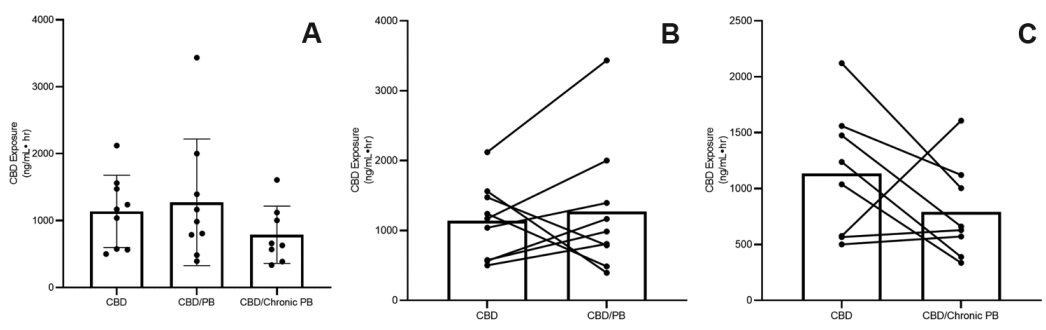

Figure 4-A-Scatterplot with bar graph of plasma CBD $A \cup C_{0-t}$ for all 9 dogs in each of 3 groups with bar representing the mean and individual $A \cup C_{0-t}$ as dots $(C B D=10 \mathrm{mg} / \mathrm{kg}$ single oral dose CBD; CBD-PB $=10 \mathrm{mg} / \mathrm{kg}$ single oral dose CBD and $4 \mathrm{mg} / \mathrm{kg}$ single oral dose PB; CBD-chronic $\mathrm{PB}=4 \mathrm{mg} / \mathrm{kg}$ oral $\mathrm{PB}$ administered twice daily for 30 days with single $10 \mathrm{mg} / \mathrm{kg} C B D$ oral dose on day 30 ). B-Beforeafter dot and bar graph of plasma CBD AUC ${ }_{0-t}$ for all 9 dogs in the CBD and CBDPB groups from phase 2 . The bar height represents the mean AUC and individual $\operatorname{dog} A \cup C_{0-t}$ are connected with a line between each group. C-Before-after dot and bar graph of plasma CBD AUC for the 8 included dogs in the CBD-chronic PB and $C B D$ groups from phase 2 and 3 . The bar height represents the mean $A \cup C_{0-t}$ and individual $\operatorname{dog} A \cup C_{0-t}$ are connected with a line between each group.

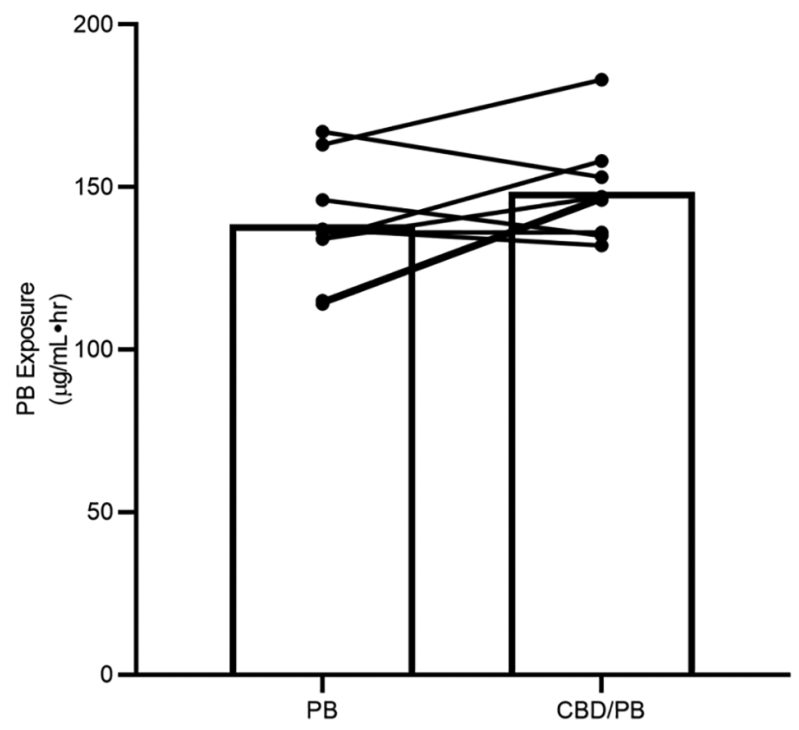

Figure 5-Before-after graph of $\mathrm{PB} A \cup \mathrm{C}_{0-\mathrm{t}}$ for all 9 dogs in both single oral $4 \mathrm{mg} / \mathrm{kg} P B$ administration and $4 \mathrm{mg} / \mathrm{kg} P B$ with concurrent $10 \mathrm{mg} / \mathrm{kg}$ CBD oral administration. The bar height represents mean $A \cup C_{0-t}$ and individual dog $A \cup C_{0-t}$ is represented by a dot, which is connected to the same dogs $A \cup C_{0-t}$ in the other treatment group from phase 2.

\section{PB PK results}

Mean $\mathrm{AUC}_{0-\mathrm{t}}$ of single-dose PB and single-dose $\mathrm{PB}$ coadministered with single-dose CBD was $138.4 \pm$ $18.3 \mathrm{ug} / \mathrm{mL} \cdot \mathrm{h}$ and $148.5 \pm 15.5 \mathrm{ug} / \mathrm{mL} \cdot \mathrm{h}$, respectively (Figure 5). There was no significant difference between groups $(P=0.13)$

All 3 dogs in the $20 \mathrm{mg} / \mathrm{kg}$ dosing group and 2 dogs in the $10 \mathrm{mg} / \mathrm{kg}$ dosing group experienced gastrointestinal signs (vomiting, $\mathrm{n}=1$; hyporexia and anorexia, $n=5)$. These signs were mild and self-limiting, lasting longest ( 7 days total) for $1 \mathrm{dog}$ in the 20 $\mathrm{mg} / \mathrm{kg}$ dosing group.

Complete blood count and pre- and postprandial bile acid concentrations were within the reference range for all dogs at all time points measured (days 0,7 ,
C and 14). Results of chemistry panel analysis were within the reference range for all dogs except 1 dog at day 0 and day 7 , that had a mild increase in ALP activity of $143 \mathrm{U} / \mathrm{L}$ (range, 15 to $140 \mathrm{U} / \mathrm{L}$ ) at day 0 and $292 \mathrm{U} / \mathrm{L}$ at day 7 . The ALP activity for all dogs at all time points are available (Supplementary Table S3). At day 14, 5 dogs had ALP activities above the reference limit of $140 \mathrm{U} / \mathrm{L}$ (range, 145 to $978 \mathrm{U} / \mathrm{L}$ ). Four of 5 dogs had ALP activities greater than twice the reference limit (mean, ALP $506 \mathrm{U} / \mathrm{L}$; range, 301 to $978 \mathrm{U} / \mathrm{L}$ ) and, therefore, considered mild AEs in accordance with the 2011 Veterinary Cooperative Oncology Group consensus. ${ }^{22}$ Two of these 4 dogs were in the $10 \mathrm{mg} / \mathrm{kg}$ dosing group and the remaining 2 were in the $20 \mathrm{mg} / \mathrm{kg}$ dosing group. There was a significant $(P<$ $0.001)$ increase in ALP activities for all doses $(5 \mathrm{mg} / \mathrm{kg}$, $10 \mathrm{mg} / \mathrm{kg}, 20 \mathrm{mg} / \mathrm{kg}$ ) and time points, except $5 \mathrm{mg} / \mathrm{kg}$ at day 7 (Table 3). There was no difference, however, between the dosing groups at any time point (Supplementary Table S4).

\section{Discussion}

To the authors' knowledge, we describe the first drug-drug interaction study between CBD and PB in healthy dogs. This 3-phased study assessed PK of 3 single oral doses of CBD administration in both a nonfasted and fasted state, single oral dose CBD (10 mg/ $\mathrm{kg}$ ) with single oral dose $\mathrm{PB}(4 \mathrm{mg} / \mathrm{kg})$, and single oral dose CBD (10 mg/kg) following chronic (30 day) oral $\mathrm{PB}$ administration $(4 \mathrm{mg} / \mathrm{kg}, \mathrm{q} 12 \mathrm{~h}$ ). Adverse events, CBC, chemistry panel analysis results, and bile acids concentrations were monitored throughout the study.

The first phase of this study evaluated the PK of 3 single CBD doses in healthy dogs during both a nonfasted and fasted state, followed by evaluation of AEs during a 2-week dosing period. There was considerable interindividual variability in the PK data in the fasted and nonfasted single-dose CBD groups of phase 1. The variability is consistent with results of previous PK studies of CBD in people and rats, which showed similar interindividual variability. ${ }^{23,24} \mathrm{~A}$ meta-analysis of CBD PK studies in humans, found variability in PK with dosage form and route, formulation, as well as with or without food. It was shown that CBD administered with food increased $\mathrm{AUC}$ and $\mathrm{C}_{\max }$. It was speculated that the presence of food would increase bile release, slowing gastric emptying, and allowing increased time for drug dissolution and absorption. ${ }^{23}$ Our study found a significant increase in $\mathrm{C}_{\max }$ in the nonfasted state of phase 1, compared with the fasted animals, but no significant elevation of the $\mathrm{AUC}_{0-\mathrm{t}}$. We speculate that our variability in CBD PK variables was in part related to differences in individual absorption, 
Table 3-Pairwise comparison of serum alkaline phosphatase activities for time at each CBD dose in healthy dogs.

\begin{tabular}{lccr}
$\begin{array}{l}\text { Contrast } \\
\text { between days }\end{array}$ & Dose $(\mathbf{m g} / \mathbf{k g})$ & $\boldsymbol{t}$ ratio & $\boldsymbol{P}$ value \\
\hline 7 and 0 & 5 & 1.04 & .5 \\
$\mathrm{I} 4$ and 0 & 5 & 5.66 & $<.001$ \\
7 and 0 & 10 & 3.59 & $<.001$ \\
$\mathrm{I} 4$ and 0 & 10 & 11.1 & $<.001$ \\
7 and 0 & 20 & 5.7 & $<.001$ \\
14 and 0 & 20 & 12.92 & $<.001$ \\
\hline
\end{tabular}

Pairwise comparison of mean alkaline phosphatase activities (U/L) from Phase I between days 0 and 7 and days 0 and $I 4$ for each of 3 CBD dosing groups $(5 \mathrm{mg} / \mathrm{kg}, 10 \mathrm{mg} / \mathrm{kg}$, and $20 \mathrm{mg} / \mathrm{kg}$ ). Significant $P$ values are bolded and existed between all time points except days 0 and 7 in the 5 $\mathrm{mg} / \mathrm{kg}$ CBD group.

body condition, variation in hepatic metabolism, as well as excretion of CBD and related to their fasted state. Based on the inherent variability and our still limited knowledge of the endocannabinoid system, it is recommended that plasma CBD levels be considered at regular intervals with chronic use.

Phases 2 and 3 of this study assessed the PK of CBD alone, with a single dose of $\mathrm{PB}$, or after 30 days of twice daily $\mathrm{PB}$ dosing. Phenobarbital, like CBD, is metabolized by the CYP450 hepatic enzymes. It induces a range of CYP450 enzymes that may also be responsible for $\mathrm{CBD}$ biotransformation, although specific information in the dog as to which CYP450 enzymes participate in biotransformation of CBD is not available. Chronic PB use has been shown to not only progressively decrease its own elimination half-life, but it has also been shown to enhance the clearance of other AEDs that undergo hepatic metabolism through CYP450 enzymes. ${ }^{2,19,21,25,26}$ For these reasons, we hypothesized that PB would also interact pharmacokinetically with CBD by increasing its biotransformation. This would result in a reduced $\mathrm{AUC}_{0-\mathrm{t}}$ and perhaps also affect $\mathrm{C}_{\max }$ if there is a large first-pass effect. However, no significant difference in the PK of CBD administered alone when compared with either single-dose coadministration of PB or with chronic PB administration was observed. It is possible that this was related to the power of the study; however, there was not a consistent change with either the chronic or single-dose PB administration. Therefore, it appears that even if an effect was missed because of sample size, the magnitude of the effect would likely be inconsequential.

When evaluating the concentration versus time curves of the 3 CBD groups in phase 2 and 3 of our study (CBD, CBD-PB, and CBD-chronic $\mathrm{PB}$ ), there was a lack of a second $\mathrm{CBD}$ concentration peak for the CBD-chronic PB group. In humans and rodents, CBD is metabolized by CYP450 hepatic enzymes; it is hydroxylated to 7-hydroxy-cannabidiol (7-OH-CBD) primarily by CYP2C19 and CYP3A4 in humans. The 7-OH-CBD undergoes further hepatic metabolism, and the metabolites are eventually excreted..$^{15}$ The CBD metabolite 7-hydroxy has also been identified when CBD was incubated with hepatic microsomes from other species (mouse, rat, guinea pig, rabbit, hamster, gerbil and cat). ${ }^{27}$ It is reasonable to assume that CYP450 biotransformation of CBD also occurs in dogs. In our CBD and CBD-PB groups, a second peak was observed in the $C$ vs $T$ curve. However, this second peak was not observed in the CBD-chronic PB group. If chronic PB administration had induced CYP450 and so increased a hepatic first-pass effect, we might have expected to see a decreased $\mathrm{C}_{\max }$ and a larger second peak. Instead, no second peak was apparent and there was no change in $\mathrm{C}_{\max }$. In addition, a second peak was not clearly observed in phase 1 at the 3 doses. It may be that the apparent second peak reflects instead a delayed absorption or may be related to the variability found in our data.

In human studies, CBD has significantly affected serum concentration of several AEDs including clobazam, topiramate, zonisamide (in adults), eslicarbazepine, and rufinamide because of CBD's inhibition of CYP450 enzymes. ${ }^{6,10,14}$ No effect was observed on serum concentrations of PB nor levetiracetam. ${ }^{14}$ However, the studies included a small sample size of individuals receiving PB and were not controlled. Phenobarbital is frequently used as a first-line AED in veterinary medicine ${ }^{2}$ and, therefore, warrants further investigation. Our study found no significant difference in the systemic exposure $\left(\mathrm{AUC}_{0-\mathrm{f}}\right)$ of single-dose $\mathrm{PB}$ compared with singledose PB coadministered with CBD. The effect of chronic coadministration of CBD and PB was not evaluated in this study, but in a pilot study involving client-owned idiopathic epileptic dogs, no significant change in serum $\mathrm{PB}$ concentration was found in the 7 dogs receiving PB after 12 weeks of CBD treatment. ${ }^{3}$ Further studies with a larger sample size are warranted to evaluate for the potential effect of chronic coadministration of $\mathrm{CBD}$ and $\mathrm{PB}$ on $\mathrm{PB}$ concentrations.

Adverse events were recorded during all phases, but only observed during phase 1 . These AEs were compared with those seen in the dogs prior to the beginning of the study. During the 6-month acclimation period (time before study's initiation), 5 dogs experience AEs including diarrhea $(\mathrm{n}=2)$, conjunctivitis $(\mathrm{n}=1)$, minor paw injury $(\mathrm{n}=1)$, and hair loss on dorsum $(n=1)$. All were minor and self-limiting in nature. During phase 1 of the study, all AEs observed were mild in nature and different from what was observed prior to the study period. During phase 1, AEs were related to gastrointestinal signs (vomiting, hyporexia, and anorexia) and an increase in serum ALP activity. Adverse events were not unexpected as adverse effects of CBD administration are common. In humans, CBD use has been associated with mild to moderate adverse effects including somnolence, poor appetite, and diarrhea, with upwards of $44 \%$ of human patients experiencing adverse effects with CBD administration., ${ }^{5,9}$ In our study, 5 of 9 dogs experienced gastrointestinal signs for a variable duration of time with the most commonly observed sign relating to inappetence. These gastrointestinal signs may be related to the delivery method (oil base). The choice of CBD administration in an oil base was made based 
on previous PK studies involving variable routes of administration in healthy Beagles in which CBDinfused oil had greater $C_{\max }$ than other delivery mechanisms. ${ }^{16}$ A placebo-controlled, cannabinoid dose escalation study involving healthy Beagles reported mild gastrointestinal AEs or lethargy accounting for $94.9 \%$ of all adverse effects recorded. Gastrointestinal AEs accounted for $65.3 \%$ and $66.3 \%$ of the adverse events recorded in the MCT oil placebo group and CBD oil group, respectively. Cannabidiol dosing in this study was found well tolerated up to approximately $62 \mathrm{mg} / \mathrm{kg} .{ }^{28}$ The adverse gastrointestinal events in our study utilizing CBD in MCT oil is consistent these previously published findings. The authors speculate that the nature of this oil base may contribute to the gastrointestinal signs seen with administration.

We administered $10 \mathrm{mg} / \mathrm{kg}$ CBD for phase 2 and phase 3 despite mild adverse effects noted during phase 1 in 2 of 3 dogs in the $10 \mathrm{mg} / \mathrm{kg}$ dosing group. Dosing of $10 \mathrm{mg} / \mathrm{kg}$ was elected based on the overall tolerability seen in dose escalation studies involving CBD in dogs where dosing approached approximately $62 \mathrm{mg} / \mathrm{kg}$ as well as based on prior PK studies of CBD in dogs. ${ }^{16,28}$

All dogs in our study had significant increase in serum ALP activity from baseline (day 0) at day 14 in all dosing groups ( $P<0.001$ for all groups). Five of 9 dogs $(55 \%)$ had increases above the upper limit of the reference range for ALP (140 U/L) at day 14 (range, 145 to $978 \mathrm{U} / \mathrm{L}$ ). Four of 5 had values greater than twice the reference limit (mean, ALP $506 \mathrm{U} / \mathrm{L}$; range, 301 to $978 \mathrm{U} / \mathrm{L}$ ) and were seen only in the $10 \mathrm{mg} / \mathrm{kg}$ and $20 \mathrm{mg} / \mathrm{kg}$ dosing groups. The most significant increase in ALP activity was observed in a dog in the $20 \mathrm{mg} / \mathrm{kg}$ dosing group, that had mild increase in ALP activity at baseline (ALP $143 \mathrm{U} / \mathrm{L}$ at day 0) and 6.8 times increase by day 14 (ALP, $978 \mathrm{U} / \mathrm{L}$ ). All other chemistry values and pre- and postprandial bile acid concentrations remained within reference range for this dog during the duration of the study and no other clinical signs were associated with this marked increase in ALP activity. There was no significant difference found in this study between dosing groups at any time point, likely due to the small sample size. It is possible that with a larger sample size, a significant difference would be seen with higher CBD dosing. No dog in this study had significant changes in any other $\mathrm{CBC}$ or chemistry value, including the liver enzyme activities of alanine transaminase and $\gamma$-glutamyl transferase. Significant increases in ALP activity have been reported in other CBD studies with chronic administration in dogs and has been reported with human use. ${ }^{9,14}$ The increase in ALP activity is likely due to the induction and inhibition of various CYP450 isoenzymes in the liver because of metabolism of CBD as is seen in humans. ${ }^{3,6,10,12-15}$ In our study, all dogs in all dosing groups had increases in ALP activity over time (between day 0 and 14). The duration of CBD administration in this study was only 14 days; it is reasonable conjecture that with continued chronic administration, ALP activities in all dosing groups would continue to increase. It is unknown if this increase would reach clinical significance in the lower dosing group $(5 \mathrm{mg} / \mathrm{kg}$ ). To evaluate clinical importance of this increase in ALP activity on liver function, pre- and postprandial bile acids were measured for all dogs at days 0,7 , and 14 of CBD administration. Pre- and postprandial bile acid concentrations remained within reference range for all dogs at all time points supporting no functional liver changes within the 14 days of administration. It is unknown if any functional changes may have occurred with a longer chronic dosing window. In light of this, the authors recommend monitoring of liver values and function with chronic CBD use, especially with concurrent drug administration, especially drugs metabolized by the liver.

The biggest limitation of this study is the small sample size in all phases and the fact that sequence randomization was not possible in all phases. In phase 1 , repeating the 3 dosage regimens in all 9 dogs would have given us greater confidence in the lack of increasing drug exposure with increased. Although a significant interaction was not observed in the PKs between CBD and PB in this report, with a larger study significance differences may have been apparent. Additionally, this study only included sexually intact female dogs so possible comparison between sex or reproductive status was not possible. A second limitation was in the duration of our dosing. In phase 1, CBD was dosed for 14 days to assess for adverse events. In a clinical setting where CBD may be used as an adjunctive AED, the use has the potential to be lifelong for the patient. Future studies where CBD is dosed daily for a longer period (several months at minimum) are needed to better assess for potential long-term adverse effects. Another limitation is the lack of control group receiving MCT oil alone; it is possible the AEs observed during phase 1 of the study may have been attributable to the oil base of the CBD. A final limitation of this study is that all dogs were young, healthy Beagles without comorbidities. Although many epileptic dogs have idiopathic epilepsy, our findings of an increase in ALP activity and gastrointestinal signs may be more pronounced in patients receiving concurrent medications. These dogs may metabolize medications differently with chronic use than healthy dogs. Future large-scale studies in epileptic dogs are warranted, preferably as prospective, double-blinded, placebo-controlled clinical trials.

The first part of our study found a lack of a significant dose effect of CBD administration on $\mathrm{C}_{\max }$ or $\mathrm{AUC}_{0-\mathrm{t}}$. Although we did observe an increase in the $\mathrm{C}_{\max }$ in nonfasted dogs, compared with fasted dogs, the $\mathrm{AUC}_{0-\mathrm{t}}$ was not affected. This may be re- 
lated to the fact that the $\mathrm{CBD}$ is administered in oil, however, it suggests that although feeding may have increased the rate of absorption, it did not increase total bioavailability. The number of dogs at each dose was limited, so these studies lack power. A larger study is indicated because if increasing dose does not increase total drug concentrations or exposure, then increasing dose may not be a useful strategy to increase efficacy. Similarly, the limited effect of feeding suggests that we do not need to be concerned with administration of CBD relative to meals. Our sample sizes are limited and interindividual variability was present, so the authors recommend the interpretation of these results with caution.

Based on the lack of significant PK interaction in this study, adjustment of the CBD dose escalation with chronic coadministration of PB does not appear indicated. Similarly, based on the single-dose administration with CBD, adjusting the dose of PB does not appear indicated. These results are consistent with our previous study where no change in serum $\mathrm{PB}$ concentrations were observed with the addition of CBD. ${ }^{3}$ Further research, however, in a larger population to assess the PK interaction of chronic concurrent $\mathrm{AED}$ use and $\mathrm{CBD}$ may reveal $\mathrm{PK}$ interactions.

\section{Acknowledgments}

Funded by Canopy Growth Corporation (Ontario, Canada), the company that provided and produced the CBD-infused MCT oil used in this study. This sponsor had no role in design, data collection, data analysis, or preparation of the manuscript.

Author Stephanie McGrath was a paid consultant for the Canopy Growth Corporation during the study period. The company did not have a role in study design, data analysis, or manuscript preparation.

The authors would like to acknowledge Dr. Ann Hess from the Colorado State Graybill Statistics Lab for her help in data analysis.

All authors contributed to the manuscript.

\section{References}

1. Monteiro R, Adams V, Keys D, Pratt SR. Canine idiopathic epilepsy: prevalence, risk factors and outcome associated with cluster seizures and status epilepticus. J Small Anim Pract. 2012;53(9):526-530.

2. Podell M, Volk HA, Berendt M, et al. 2015 ACVIM small animal consensus statement on seizure management in dogs. $J$ Vet Intern Med. 2016;30(2):477-490.

3. McGrath S, Bartner LR, Rao S, Packer RA, Gustafson DL. Randomized blinded controlled clinical trial to assess the effect of oral cannabidiol administration in addition to conventional antiepileptic treatment on seizure frequency in dogs with intractable idiopathic epilepsy. $J$ Am Vet Med Assoc. 2019;254(11):1301-1308.

4. Volk HA, Matiasek LA, Feliu-Pascual AL, Platt SR, Chandler KE. The efficacy and tolerability of levetiracetam in pharmacoresistant epileptic dogs. Vet J. 2008;176(3):310-319.

5. Press CA, Knupp KG, Chapman KE. Parental reporting of response to oral cannabis extracts for treatment of refractory epilepsy. Epilepsy Behav. 2015;45:49-52.

6. Geffrey AL, Pollack SF, Bruno PL, Thiele EA. Drug-drug interaction between clobazam and cannabidiol in children with refractory epilepsy. Epilepsia. 2015;56(8):1246-1251.

7. Jones NA, Glyn SE, Akiyama S, et al. Cannabidiol exerts anticonvulsant effects in animal models of temporal lobe and partial seizures. Seizure. 2012;21(5):344-352.

8. Consroe P, Wolkin A. Cannabidiol-antiepileptic drug com- parisons and interactions in experimentally induced seizures in rats. J Pharmacol Exp Ther. 1977;201(1):26-32.

9. Miziak B, Walczak A, Szponar J, Pluta R, Czuczwar SJ. Drugdrug interactions between antiepileptics and cannabinoids Expert Opin Drug Metab Toxicol. 2019;15(5):407-415.

10. Anderson LL, Absalom NL, Abelev SV, et al. Coadministered cannabidiol and clobazam: preclinical evidence for both pharmacodynamic and pharmacokinetic interaction. Epilepsia. 2019;60(11):2224-2234.

11. Patra PH, Barker-Haliski M, White HS, et al. Cannabidiol reduces seizures and associated behavioral comorbidities in a range of animal seizure and epilepsy models. Epilepsia. 2019;60(2):303-314.

12. Brutlag A, Hommerding H. Toxicology of marijuana, synthetic cannabinoids, and cannabidiol in dogs and cats. Vet Clin North Am Small Anim Pract. 2018;48(6):1087-1102.

13. Bornheim LM, Everhart ET, Li J, Correia MA. Characterization of cannabidiol-mediated cytochrome $\mathrm{P} 450$ inactivation. Biochem Pharmacol. 1993;45(6):1323-1331.

14. Gaston TE, Bebin EM, Cutter GR, Liu Y, Szaflarski JP, UAB CBD Program. Interactions between cannabidiol and commonly used antiepileptic drugs. Epilepsia. 2017;58(9):1586-1592.

15. Lucas CJ, Galettis P, Schneider J. The pharmacokinetics and pharmacodynamics of cannabinoids. Br J Clin Pharmacol. 2018;84(11):2477-2482.

16. Bartner LR, McGrath S, Rao S, Hyatt LK, Wittenburg LA. Pharmacokinetics of cannabidiol administered by 3 delivery methods at 2 different dosage to healthy dogs. Can J Vet Res. 2018;82(3):178-183.

17. Wagner JG. Pharmacokinetics for the Pharmaceutical Scientist. CRC Press; 1993.

18. Motulsky HJ, Brown RE. Detecting outliers when fitting data with nonlinear regression - a new method based on robust nonlinear regression and the false discovery rate. BMC Bioinformatics. 2006;7:123.

19. Al-Tahan F, Frey HH. Absorption kinetics and bioavailability of phenobarbital after oral administration to dogs. $J$ Vet Pharmacol Ther. 1985;8(2):205-207.

20. Pedersoli WM, Wike JS, Ravis WR. Pharmacokinetics of single doses of phenobarbital given intravenously and orally to dogs. Am J Vet Res. 1987;48(4):679-683.

21. Ravis WR, Pedersoli WM, Wike JS. Pharmacokinetics of phenobarbital in dogs given multiple doses. Am J Vet Res. 1989;50(8): 1343-1347.

22. Veterinary cooperative oncology group - common terminology criteria for adverse events (VCOG-CTCAE) following chemotherapy or biological antineoplastic therapy in dogs and cats v1.1. Vet Comp Oncol. 2016;14(4):417-446.

23. Lim SY, Sharan S, Woo S. Model-based analysis of cannabidiol dose-exposure relationship and bioavailability. Pharmacotherapy. 2020;40(4):291-300.

24. Zgair A, Wong JC, Lee JB, et al. Dietary fats and pharmaceutical lipid excipients increase systemic exposure to orally administered cannabis and cannabis-based medicines. Am J Transl Res. 2016;8(8):3448-3459.

25. Muñana KR, Nettifee-Osborne JA, Papich MG. Effect of chronic administration of phenobarbital, or bromide, on pharmacokinetics of levetiracetam in dogs with epilepsy. $J$ Vet Intern Med. 2015;29(2):614-619.

26. Forrester SD, Wilcke JR, Jacobson JD, Dyer KR. Effects of a 44-day administration of phenobarbital on disposition of clorazepate in dogs. Am J Vet Res. 1993;54(7):1136-1138.

27. Harvey DJ, Brown NK. Comparative in vitro metabolism of the cannabinoids. Pharmacol Biocbem Bebav. 1991;40(3):533-540.

28. Vaughn D, Kulpa J, Paulionis L. Preliminary investigation of the safety of escalating cannabinoid doses in healthy dogs. Front Vet Sci. 2020;7:51.

\section{Supplementary Materials}

Supplementary materials are posted online at the journal website: avmajournals.avma.org. 